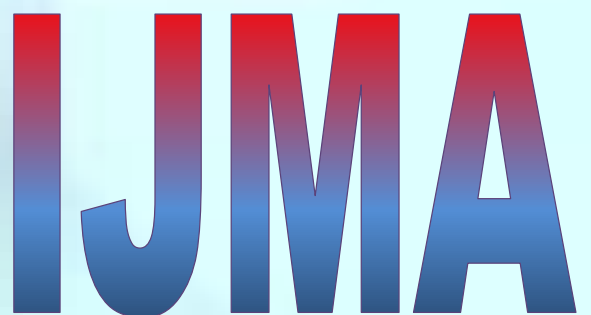

INTERNATIONAL

Journal of MEdical

\section{ARTS}

Volume 3, Issue 1 (Winter 2021)

http://ijma.journals.ekb.eg/

Print ISSN: 2636-4174

Online ISSN: 2682-3780

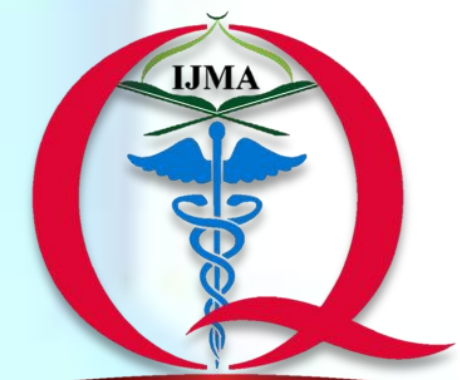

International Jounnal of Medical Arts

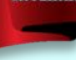




\section{About IJMA}

- International Journal of Medical Arts is the Official Journal of the Damietta Faculty of Medicine, Al-Azhar University, Egypt

- The First Issue was published in July 2019

- It is an International, Open Access, Double-blind, Peerreviewed Journal

- Published four times a year

- Published under the following license: Creative Commons Attribution-ShareAlike 4.0 International Public License (CC BY-SA 4.0). It had updated from the Creative Commons license [CC BY] in volume 2, Issue 4, October 2020

- The Egyptian Knowledge Bank hosts the web site of IJMA

- The Egyptian Knowledge Bank supports IJMA

- IJMA is indexed in the "Directory of Open Access Journals" Indexed on 15 January 2021.

- IJMA follows the regulations of the International Committee of Medical Journal Editors (list date 1/21/20)

- IJMA is a member of The International Society of Managing and Technical Editors

- IJMA is listed in Index Copernicus

- IJMA is listed in Publons, as EKB is an official partner with Clarivate Analytics
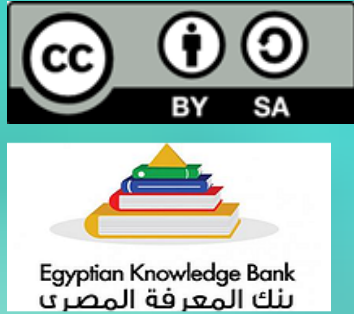

\section{DOAJ}

CMNE

ISMTE

INDEX COPERNICUS

publons 
International Journal of Medical Arts 2021; 3 [1]: 1152-1162.

Available online at Journal Website
https:/lijma.journals.ekb.eg/
Main subject [General Surgery]

Original article

\title{
Self-gripping Mesh in Inguinal Hernia Repair
}

\author{
Ibrahim Goda Ibrahim Atia[1]; Amr Abd-Alghany Sarhan[2]; Mohamed Mustafa Balbola[2]
}

Dekirnis Central Hospital, Ministry of Health, Mansoura, Dakahlia, Egypt[1]

Department of General Surgery, Damietta Faculty of Medicine, Al-Azhar University, Egypt[2]

Corresponding author: Ibrahim Goda Ibrahim Atia

Email: dr.ibrahimgoda@gmail.com

Submission date: November 30, 2020; Revision date: January 06, 2021; Acceptance date: January 07, 2021

DOI: 10.21608/ijma.2021.50241.1214

\section{ABSTRACT}

Background: Inguinal hernia repair is a common procedure that general surgeons undertake with different techniques aiming for a better outcome.

Aim of the work: Comparison between self-gripping mesh and sutures for mesh fixation in open [Lichtenstein] inguinal repair.

Patients and Methods: This study is a prospective trial. Fifty adult males scheduled for inguinal hernia repair were classified into two groups: group $A[n=25]$ underwent inguinal repair with self-gripping mesh; group $B[n=25]$ underwent inguinal repair with mesh fixation using proline sutures.

Results: The mean operative time was $37.4 \pm 13.5$ and $48.81 \pm 14.7$ minutes in groups $A$ and $B$, respectively. The incidence of seroma, scrotal edema, and wound infection was $4 \%, 8 \%$, and $8 \%$ in the group $[A]$ compared to $12 \%, 24 \%, 12 \%$ in the group $[B]$, respectively. No recurrence was reported in either group. However, $12 \%$ and $80 \%$ in groups $A$ and $B$ had mild postoperative pain $[p<0.001]$. After three months of surgery, no cases of chronic groin pain reported in the group $[A]$ compared to three in the group $[B][p>0.05]$. The mean time to return to normal activities in a group $[A]$ was 2.51 days and 5.97 days in the group $[B][p<0.001]$. Mean pain visual analogue scale [VAS] scores at 6 months were 0.5 [0.4 to 1.5] in the self-gripping mesh group and 8.6 [3.3 to 12.5] in sutured mesh group [ $p<0.01]$. The mean cost of ProGrip mesh was higher than the mean cost of polypropylene mesh [4508 VS 555 LE] [p<0.001].

Conclusion: Lichtenstein open inguinal hernia repair with ProGrip low-density polyester mesh is a safe, with short operative time, simple and effective method for inguinal hernia repair and may improve patient general health, reduce postoperative pain and improve quality of life. However, its high price makes its use questionable in low economic settings.

Keywords: Inguinal Hernia; Hernia Repair; ProGrip Mesh; Chronic Groin Pain; Self-gripping Mesh.

This is an open-access article registered under the Creative Commons, ShareAlike 4.0 International license [CC BY-SA 4.0] [https://creativecommons.org/licenses/by-sa/4.0/legalcode.

Please cite this article: Atia IGI, Sarhan AA, Balbola MM. Self-gripping Mesh in Inguinal Hernia Repair. IJMA 2021; 3[1]: 1152-1162. DOI: 10.21608/ijma.2021.50241.1214

* Main subject and any subcategories have been classified according to the research topic. 


\section{INTRODUCTION}

Inguinal hernias are common problems that face surgeons, representing about $10-12 \%$ of all surgeries. An inguinal hernia can be defined as the protrusion of a part or whole abdominal viscous into the inguinal canal, either through the deep ring or through Hasselbach's triangle. About $75 \%$ of hernias are found in the groin, with a lifetime risk of $27 \%$ in men and $3 \%$ in women. About one-third of inguinal hernias are direct, while the rest are indirect[1]. Inguinal hernia repair is one of the most frequent procedures a general surgeon performs. Various surgeons have contributed to different techniques for a better outcome in relation to different complications like groin pain, infection, testicular atrophy and recurrence etc., related to older techniques ${ }^{[2]}$.

Recently, the gold standard for hernia repair has become using mesh procedure in hernia surgery, which led to a substantial decrease in hernia recurrence rate[1]. Using mesh has reduced recurrence rates to below $5 \%$ in repairing the inguinal hernia, and postoperative morbidity is now often attributed to other outcome measures, especially chronic pain and surgical site infection [SSI] [3]. Mesh fixation includes tacks or staples, sutures, self-fixing meshes, fibrin, or other glues. However, among surgeons, there is no consensus on the best surgical technique. The surgeon's personal preference decides which mesh fixation method to be used[1].

Heavyweight polypropylene meshes can elicit inflammatory reactions responsible for mesh shrinkage when scar tissue evolves. Thus, using low-weight meshes has been recommended to limit the extent of fixation. Therefore, to avoid the need for additional fixation, self-gripping meshes have been developed[4]. Authors advocating sutureless mesh hernioplasty suggest that they lower tension in suture line with better leveling that results in a rapid embodiment of mesh without the formation of dead space, which reduces chances of nerve entrapment and post-operative complications and improves postoperative recovery. However, some studies reported more chances of displacement, migration, and folding of self-gripping mesh in hernia repair than the traditional Lichtenstein technique, causing failure of the whole procedure[3]. However, there is still a debate about the criteria of an ideal mesh and the optimal fixation technique to prevent postoperative pain. The most supported theories for Chronic groin pain [CGP] after hernioplasty are inguinal nerves entrapment by sutures and foreign body inflammatory reaction induced by the alloplastic mesh or fixation sutures ${ }^{[4]}$.

A prosthetic mesh has certain features like elasticity, density, material, strength, and pore size. Standard heavyweight polypropylene mesh is the most frequently used one because it is affordable, available in most hospitals, inert, non-absorbable, and has tensile strength enough to prevent the recurrence. Nevertheless, there are actual problems with mesh usage as foreign body sensation at the site of surgery and chronic postoperative pain or discomfort. This created a disagreement about standard heavyweight polypropylene mesh. Mesh made of Polyester might be an appropriate alternative, but it is not popular as polypropylene mesh. Newer lightweight meshes have been manufactured to overcome those problems [1].

\section{AIM OF THE WORK}

This study aimed to compare between selfgripping mesh and sutures for mesh fixation in open [Lichtenstein] inguinal repair with an assessment of operative time, intraoperative complications [primary, secondry], hospital stay, return to normal life activity, post-operative pain, and costs.

\section{PATIENTS AND METHODS}

This prospective study included 50 adult male cases scheduled for inguinal hernia repair at AlAzhar University Hospital- New Damietta, department of surgery, who met the inclusion criteria in the period between November 2019 and October 2020. Approval by the ethical committee for research in Al-Azhar university faculty of medicine [Damietta] was obtained before initiating this study. Details of the operation technique and complications were explained to the patients, and informed written consents were obtained.

Patient's sampling method was the convenience sampling method. Patients were randomized using simple randomization of flipping a coin into two 
groups according to the type of surgery: Group [A] [self-gripping mesh]: 25 patients had inguinal repair with self-gripping mesh; Group [B]: [sutures for mesh fixation]: 25 patients had inguinal repair with mesh fixation using proline sutures.

Patients were selected based on the following criteria: All adult male patients who attended the surgical outpatient department with inguinal hernia direct, indirect, or pantaloons type. On the other side, patients were excluded from this study based on the following criteria: Patients aged below 18 and above 65 years old. Patients with a complicated inguinal hernia that needed urgent surgical intervention. Patients with recurrent inguinal hernia. Patients with urological complaints such as dysuria or urine retention. Patients with chronic chest problems causing cough such as chronic obstructive pulmonary disease [COPD]. High-risk patients according to the American Society of Anesthesiologists [ASA]. Suspected intraabdominal malignancy on ultrasonography and subsequently computed tomography [CT] basis and patients who refused to participate in this study.

All participants were subjected to history taking as personal, past, and family history with particular stress upon hernia's risk factors as gender, smoking, heavy weight lifting and the nature of patient's work, chronic cough and COPD, dysuria, chronic constipation, and benign prostatic hyperplasia. Patients had a clinical examination in the form of general and local examination with particular stress upon other hernia orifices, organomegaly, and cardiopulmonary status.

Patients were subjected to routine laboratory investigations including complete blood analysis, complete urine analysis, blood urea, and serum creatinine, alanine transaminase [ALT] and aspartate transaminase [AST], prothrombin time $[P T]$, partial thromboplastin time [PPT], international normalization ratio [INR] and viral markers [HBsAg and HCV IgG]. Patients were subjected to radiological investigations in the form of ultrasonography [US] of the abdomen and scrotum, computed tomography [CT] scan for any suspected pathology on ultrasonography, and chest X-ray.

All patients were subjected to treatment as follow:
Patients' preoperative preparations were done by taking a shower with an antiseptic solution the night before surgery and/or the morning of surgery. Hairy patients were advised to remove hair. Low doses of low molecular weight heparin, enoxaparin sodium [Clexane $1 \mathrm{mg} / \mathrm{kg}$ subcutaneously] was given for high-risk patients.

Prophylactic antibiotics: Third-generation cephalosporin [cefotaxime] $1 \mathrm{gm}$ by intravenous drip one dose preoperative and another dose after 2hours postoperative. Foley catheters were installed for patients with large hernia sac or those with a complete inguinoscrotal hernia. Identification of the hernia side was achieved with a marker pen.

\section{Operative details:}

Operations were performed under spinal or general anesthesia with the patients in the supine position. Skin disinfection was done by $10 \%$ Povidone-iodine antiseptic solution.

Operative technique: The skin incision was made half-inch above the medial two-thirds of the inguinal ligament to expose the external oblique aponeurosis, then it was divided. The cord is dissected out of its bed to obtain a suitable space for mesh positioning under the external oblique aponeurosis. Identification and preservation of the ilioinguinal, iliohypogastric, and genital branch of the genito-femoral nerve were done. Dissection of the hernia sac from the spermatic cord was gently done. In the case of an indirect oblique hernia, the sac was dissected from the cord till its proper neck; then, the sac was excised after transfixing-ligation of its neck by absorbable suture material [Vicryl 0]. In the case of a direct hernia with weak fascia transversalis, the sac was reduced without opening.

\section{Mesh fixation:}

Group A: A 6 X11 cm progrip $®$ mesh was laid over the posterior wall of the inguinal canal, and tailoring of the mesh was done to accommodate the created space overlapped the pubic tubercle minimally by $1 \mathrm{~cm}$. The fixation procedure was performed by applying pressure on the mesh, beginning medially at the pubic bone, then laterally onto the conjoint tendon. The cranial part of the mesh was fixed below the external oblique aponeurosis and the spermatic cord passed via a 
slit in the mesh. The size of the slit was adapted to the diameter of the cord and the encircling anatomical structures. After that, the mesh was driven down in the direction of the inguinal ligament. Care was taken so one can avoid folding the mesh. To assist the mesh positioning, one suture of absorbable suture was taken to fix it to the pubic tubercle.

Group B: A sheet of polypropylene [PP] mesh cut to shape and located over the posterior wall of the inguinal canal, so it overlapped the pubic tubercle by at least I $\mathrm{cm}$ medially. Fixation of the mesh was achieved by an interrupted polypropylene $2 / 0$ suture to the inguinal ligament inferiorly and interrupted sutures to the conjoint area superiorly. A slit was cut for the spermatic cord and the tails secured again collectively with permenant sutures, as described by Lichtenstein in 1984.

Closure: After insertion of the suction drain, the external oblique aponeurosis was then closed anterior to the spermatic cord structures by a nonabsorbable suture. The subcutaneous tissue was approximated by an absorbable suture [vicryl 2/0], and skin was closed by non-absorbable interrupted sutures.

Postoperative management: Analgesia and mild sedative were important in the immediate postoperative period for a smooth recovery from anesthesia without pain or irritability to avoid any stress on the suture line, leading to separation of the sutures. All patients were nursed in a semisitting position in bed. Immediate ambulation was done for patients to get out of bed, the first day postoperative. Wound suction was discontinued if the drainage ceases [minimal amount 20-30 cc/ day] at least 6 - 7 days postoperative. Sutures were taken out the $10^{\text {th }}-12^{\text {th }}$ day postoperative.

Diet and discharge: Patients were allowed to have liquid fluids and semi-solid food in the evening of the day of surgery after hearing good peristalsis, and they were discharged once they tolerate oral feeding.

Follow up: All patients were followed up during hospital stay and 7 days after discharge; 1 month; 3 months; and a last evaluation 6 months after surgery [physical examination by surgeon]. On every follow-up visit, the patient was well assessed clinically for the presence of signs of; early postoperative complications [within 30 days postsurgery] [e.g., local inflammation, swelling as seroma, recurrence or tenderness, hematoma, wound infection, and pain], and for late postoperative complications [30-day post-surgery] [e.g., chronic groin pain, mesh infection or testicular atrophy]. Patients were subjected to ultrasonographic study at the $6^{\text {th }}$ month postoperatively to check the stability and integrity of the mesh or excessive scarring.

Methods of evaluation: The surgeon reported technical difficulties as in cases of disturbed anatomy, also operative time, and mesh fixation time. The patient reported postoperative pain using a visual analogue scale [VAS], and then the needed analgesic dose to kill pain was recorded. Also, the patient reported the time needed to return to normal activity and patient satisfaction. Seroma, wound infection [superficial/deep], recurrence, hematoma, and mesh infection were detected clinically and confirmed by the US. Mesh shrinkage, thickening of the spermatic cord, and testicular atrophy were assessed by the US.

The visual analogue scale [VAS] was selfcompleted by the patient. The patients were requested to set a line perpendicular to the VAS line at the point that shows their pain intensity. Using a ruler, the score is calculated by measuring the distance $[\mathrm{mm}]$ on the $10 \mathrm{~cm}$ line between the "no pain" point and the patient's point, giving a range of scores from 0-100. The following cut points on the pain VAS have been recommended: none [0-4mm], mild [5-44mm], moderate [45-74mm] and severe $[75-100 \mathrm{~mm}]^{[5]}$.

Statistical analysis: Data has been fed to the computer and analyzed using IBM SPSS software package version 20.0 [statistical package for social sciences, Armonk, NY: IBM Corp]. Continuous data were demonstrated in the form of mean $\pm S D$, while categorical data were demonstrated in count and percent. Comparisons of continuous data were performed utilizing the student $t$-test, while categorical data were done using the Chi-square test. A P-value less than 0.05 was considered 
statistically significant.

\section{RESULTS}

In the present study, the mean age of patients was 46.9 years in the group [A] compared to 47.4 years in the group $[B]$. In group $[A]$, the mean $B M I$ was $29.4 \pm 4.13 \mathrm{Kg} / \mathrm{m}^{2}$ compared to $29.5 \pm 2.9 \mathrm{Kg} / \mathrm{m}^{2}$ in the group [B]. 7 cases [28\%] in the group [A] and 6 cases [24\%] in the group [B] were smokers and had chest problems. 2 patients [8\%] in the group [A] and one patient $[4 \%]$ in the group $[B]$ had diabetes. There were no significant statistical differences between both groups regarding basic demographic and clinical data [ $p>0.05]$ [Table-1].

In group $[A]$, operative time ranged from $35-55$ minutes with a mean of $37.4 \pm 13.5$ minutes. However, in the group [B], it ranged from 50- 75 minutes with a mean of $48.81 \pm 14.7$ minutes $[p<0.001]$. The mean time needed for mesh fixation in group [A] was $5.13 \pm 1.09$ [range 3-7] minutes, compared to $15.3 \pm 1.49$ [range 13-18] minutes in group [B] $[p<0.001]$ [Table-2].

The incidence of seroma was 1 patient [4\%] in group [A] and 3 patients [12\%] in group [B]. 2 cases [8\%] in group $[A]$ and 6 cases [24\%] in group [B] developed mild scrotal edema. wound infection occurred in two cases in the group $[A]$ and four cases in the group $[B][p>0.05]$. No recurrence occurred in either group [Table-3].

Three patients $[12 \%]$ in the group $[A]$ and 20 patients [80\%] in the group $[B]$ had mild pain postoperatively that required increasing the dose of analgesia $[p<0.001]$. While no reported cases of chronic groin pain in the group $[A]$, there were 3 cases of chronic pain in the group $[B]$ that had mild pain after three months of surgery [ $p>0.05]$. The mean VAS scores after surgery were 32.7 in the $[A]$ group and 61.2 in the $[B]$ group $[P<0.001]$. Mean time to return to normal activities in group $A$ was 2.51 days and 5.97 days in the group $[B][P<0.001]$. Overall, both groups of patients were satisfied with their surgical experience [Table-4].

Patients in the self-gripping mesh group had consistently lower VAS scores at all follow-up sessions with statistically significant differences from sutured mesh group. Mean VAS pain scores at 6 months were 0.5 [0.4 to 1.5] in self-gripping mesh group and 8.6 [3.3 to 12.5] in sutured mesh group [ $p<0.01]$ [Table-5].

Table [1]: Baseline and demographic data in study groups

\begin{tabular}{|c|c|c|c|}
\hline & $\begin{array}{c}\text { Group [A] } \\
{[n=25]}\end{array}$ & $\begin{array}{c}\text { Group [B] } \\
{[n=25]}\end{array}$ & $P$ value \\
\hline Age [years] & $46.9 \pm 12.2$ & $47.4 \pm 10.9$ & 0.877 \\
\hline Body mass index $\left[\mathrm{kg} / \mathrm{m}^{2}\right]$ & $29.4 \pm 4.13$ & $29.5 \pm 2.9$ & 0.837 \\
\hline \multicolumn{3}{|l|}{ Occupation } & \multirow[t]{4}{*}{0.697} \\
\hline Non-manual work & $8[32 \%]$ & $9[36 \%]$ & \\
\hline Manual work & $7[28 \%]$ & $7[28 \%]$ & \\
\hline Unemployed & 10 [40\%] & $9[36 \%]$ & \\
\hline Smoker & $7[28 \%]$ & 6 [24\%] & 0.891 \\
\hline Co-morbidity [COPD] & $1[4 \%]$ & $2[8 \%]$ & 0.822 \\
\hline Diabetes mellitus & $2[8 \%]$ & $1[4 \%]$ & 0.821 \\
\hline Cardiac disease & $5[20 \%]$ & $4[16 \%]$ & 0.720 \\
\hline Previous surgery & $3[12 \%]$ & $4[16 \%]$ & 0.899 \\
\hline Hernia side & & & 0.572 \\
\hline Right & 16 [64\%] & 14 [56\%] & \\
\hline Left & $9[36 \%]$ & $11[44 \%]$ & \\
\hline Hernia classification & & & 0.769 \\
\hline Indirect & $14[56 \%]$ & 16 [64\%] & \\
\hline Direct & 9 [36\%] & $8[32 \%]$ & \\
\hline Combined & $2[8 \%]$ & $1[4 \%]$ & \\
\hline
\end{tabular}


Table [2]: Operative data in study groups

\begin{tabular}{|c|c|c|c|}
\hline & Group $[A][n=25]$ & Group [B] [n= 25] & $P$ value \\
\hline \multicolumn{3}{|l|}{ Anesthesia type } & \multirow[t]{3}{*}{0.750} \\
\hline Spinal anaesthesia & $22[88 \%]$ & $23[92 \%]$ & \\
\hline General anaesthesia & $3[12 \%]$ & $2[8 \%]$ & \\
\hline Heparin [Clexane $1 \mathrm{mg} / \mathrm{kg}$ s.c.] preoperative & $5[20 \%]$ & $4[16 \%]$ & 0.781 \\
\hline Duration of surgery [min] & $37.4 \pm 13.5$ & $48.8 \pm 14.1$ & $<0.001$ \\
\hline \multicolumn{3}{|l|}{ Difficulty of dissection } & \multirow[t]{3}{*}{0.851} \\
\hline Normal & $20[80 \%]$ & $21[84 \%]$ & \\
\hline More difficult & $5[20 \%]$ & $4[16 \%]$ & \\
\hline Mean time needed for mesh fixation & $5.13 \pm 1.09$ & $15.3 \pm 1.49$ & $<0.001$ \\
\hline Mean cost of mesh [LE] & $4508 \pm 26.2$ & $555 \pm 25.6$ & $<0.001$ \\
\hline
\end{tabular}

Table [3]: Postoperative complications and hospital stay in study groups

\begin{tabular}{|l|c|c|c|}
\hline & Group [A] [n= 25] & Group [B] [n= 25] & P-value \\
\hline Seroma & $1[4 \%]$ & $3[8 \%]$ & 0.253 \\
\hline Hematoma & $1[4 \%]$ & $2[8 \%]$ & 0.556 \\
\hline Surgical-site infection & $2[8 \%]$ & $4[8 \%]$ & 0.174 \\
\hline Scrotal edema & $2[8 \%]$ & $6[24 \%]$ & 0.123 \\
\hline Hospital stay [days] & $1.5 \pm 0.5$ & $1.6 \pm 0.3$ & 0.452 \\
\hline
\end{tabular}

Table [4]: Postoperative pain and quality of life between study groups

\begin{tabular}{|l|c|c|c|}
\hline & Group [A] [n= 25] & Group [B] [n= 25] & P-value \\
\hline Mean VAS scores before surgery & 25.7 & 23.9 & 0.497 \\
\hline Mean Postoperative pain on VAS scores & $32.7 \pm 10.6$ & $61.2 \pm 14.1$ & $<0.001$ \\
\hline Patient satisfaction & $1[4 \%]$ & $3[12 \%]$ & $<0.505$ \\
$\quad$ Not satisfied & $24[96 \%]$ & $22[88 \%]$ & \\
$\quad$ Satisfied & $2.51 \pm 0.64$ & $5.97 \pm 3.71$ & $<0.001$ \\
\hline Return to normal activity [days] & $3[12 \%]$ & $20[80 \%]$ & $<0.001$ \\
\hline Need for analgesia After surgery & $0[0]$ & $3[12 \%]$ & 0.123 \\
\hline Chronic pain & & & \\
\hline
\end{tabular}

Table [5]: Follow up of study groups according to pain [0-100]

\begin{tabular}{|l|c|c|c|}
\hline & Group [A] [n= 25] & Group [B] [n= 25] & P value \\
\hline First day & $32.7 \pm 10.6$ & $61.2 \pm 14.1$ & $<0.001$ \\
\hline One week & $24.5 \pm 12.3$ & $54.5 \pm 18.9$ & $<0.001$ \\
\hline One month & $8.5 \pm 12.3$ & $35.5 \pm 21.6$ & $<0.001$ \\
\hline Three months & $1.1 \pm 2.3$ & $26.3 \pm 24.3$ & $<0.001$ \\
\hline Six months & $0.5 \pm 0.8$ & $8.6 \pm 11.3$ & 0.007 \\
\hline
\end{tabular}




\section{DISCUSSION}

The self-gripping mesh is a revolutionary modification as without sutures, it can be secured, avoiding the risk for nerve entrapment and preserving anatomical structures relationship[6]. Also, the resorbable polyactic acid micro-grips of the sutureless mesh are more blunt to avoid damage to surrounding tissues, including delicate ductus deferens and nerve fibers ${ }^{[7,8]}$.

Progrip $®$ mesh is developed to avoid suture fixation and also to diminish the formation of excessive fibrosis during healing[9]. The microgrips are club-shaped 1-mm projections that are made of biodegradable polylactic acid [PLA]. The microgrips integrate into the tissue below the lower rim of the mesh for $0.5 \mathrm{~mm}$ and produce stronger tissue incorporation at 5 days than fixation by staples ${ }^{[10]}$.

This study has been designed to evaluate selfgripping mesh's outcome versus the conventional sutured mesh in repairing the inguinal hernia. In group [A] [sutureless mesh], the mean ages of patients were 46.9 years, while, In group [B] [sutures for mesh fixation], the mean ages of patients was 47.4 years with no significant difference statistically between both groups regarding age $[p=0.877]$. This is similar to the mean age at repair as reported by Maharaul et al.[11] and Verhagen et al.[9].

This study shows that in-group $[A]$, the mean BMl was $29.4 \pm 4.13 \mathrm{Kg} / \mathrm{m}^{2}$, and in the group [B], $29.5 \pm 2.9 \mathrm{Kg} / \mathrm{m} 2$. These results are comparable with the findings of Ceith et al. ${ }^{[12]}$ and Verhagen et al. ${ }^{[9]}$.

In the current study, 7 cases [28\%] in the group $[A]$ and 6 cases $[24 \%]$ in the group $[B]$ were smokers and had chest problems. Verhagen et al.[9] reported $28 \%$ of patients in the self-gripping mesh group and $23.75 \%$ in sutured mesh group were smokers and had chest problems. Kingsnorth et al. ${ }^{[10]}$ reported that $27.9 \%$ of cases were smokers and had chest problems. In their study, Wang and Zhang[13] reported that $57.4 \%$ in the sutureless mesh group and $29.2 \%$ in sutured mesh group were smokers and had chest problems.

In the current study, the type [direct or indirect] and/or the site [right, left or bilateral] of hernia in the groin did not affect the procedure. Wang and Zhang [13], in their study, found that inguinal hernia was more common on the right-sided [67\%] than leftsided hernia [33\%], which agrees with our results as we found the predominant type of right inguinal hernia. All patients in our study underwent an operation by an anterior approach using mesh without any intraoperative complications.

As regard co-morbidity, this study shows that 2 patients [8\%] in the group [A] and one patient [4\%] in the group $[\mathrm{B}]$ were diabetics. This is comparable with the results of Kingsnorth et al., who reported that $6 \%$ of their cases were diabetics[10].

Regarding the operative time [calculated from skin incision to skin closure], in the group [A], it ranged from 35-55 minutes with a mean of $37.4 \pm 13.5$ minutes. However, in the group $[B]$, it ranged from 50- 75 minutes with a mean of 48.81 \pm 14.7 minutes, with an increase in the operative time ranged 15-20 minutes. The operative time in the group $[B]$ was statistically significantly longer than in group $[A][p<0.001]$.

Regarding the time needed for mesh fixation, in the group $[A]$, it ranged from 3-7 minutes with a mean of $5.13 \pm 1.09$ minutes, whereas in the group $[B]$, it ranged from 13-18 minutes with a mean of $15.3 \pm 1.49$ minutes. The mesh fixation time was shorter in the group $[A][p<0.001]$. These results are consistent with Batabyal et al. ${ }^{[14]}$ and Maharaul et al. [11].

The incidence of seroma in our study was 1 patient $[4 \%]$ in the group $[A]$ and 3 patients $[12 \%]$ in the group $[B]$. All 4 patients who developed mild seroma had an indirect inguinoscrotal hernia with a larger sac that required more distal dissection, creating a large surface area and large dead space that may cause seromas formation. These seromas resolved spontaneously without any intervention in all cases during the first few [3-9] post-operative days.

In the present study, 2 cases [8\%] in the group [A] and 6 cases [24\%] in the group [B] developed mild scrotal edema that resolved spontaneously during the few post-operative days. At the same time, Batabyal et al. [14] reported that scrotal edema was $2 \%$ in the self-gripping mesh group. 
In the present study, wound infection occurred in 2 cases in the group $[A]$ and 4 cases in the group $[B]$ during the first few postoperative [4-7] days that resolved on antibiotics. In our study, over the short period of follow-up, we reported no recurrence in either group. Therefore, long-term follow-up can judge the recurrence as some studies said that if recurrence to occur, most incidence supposed to be within 2 years after operation [15].

A previous study suggested that decreased operative time could decrease the rate of wound infection in self-gripping mesh repair as it reduces the exposure time of the open wound[10]. However, in the current study, the infection rates were similar in both study groups. These results may be explained by the fact that many factors have been responsible for the increased infection risk after hernia repairs, such as the patient's age, sex, the type of hernia, mesh, and repair technique[16].

Anadol et al. reported that sutureless mesh could be better than sutured mesh regarding recurrence as the microhooks permit adherence of the mesh to the whole surface of the underlying structures, providing a watertight repair. On the other hand, the sutured mesh has a decreased contact with underlying structures, increasing the risk of mesh migration for an indefinite time[17].

Tarchi and his colleagues reported no intraoperative complications in their study. Early postoperative comorbidities are in the form of hematoma, seroma $[5.7 \%]$, superficial wound infection [1\%], retention of urine [0.5\%], and scrotal swelling [1\%], which was in agreement with our study in both groups [18].

In our study, there was no significant statistical difference between both groups regarding complications despite the increased incidence of complications in sutured group than in self-gripping mesh groups. Lin and his coworker's assessed mesh fixation with glue versus suture found that hematomas incidence was higher significantly in the sutured mesh group than in the glue fixation group. However, they reported no significant difference between the two groups regarding early recurrence, wound infection, mesh infection, late recurrence, and late chronic pain ${ }^{[19]}$.
In addition, Sun and his colleagues found in their study that self-grip meshes were superior to sutured meshes regarding the duration of the operation, hematoma, and recovery time to daily activities. There were no significant differences between the two groups for superficial wound infection, mesh/ deep infection, seroma, persisting numbness, and postoperative length of hospital stay which was in agreement with our results[ ${ }^{[20]}$.

Rönkä and his coworkers in their study found that the duration of operation was $32 \pm 9$, and $38 \pm 9$ minutes in the self-gripping, and suture groups, respectively. They reported no significant postoperative differences in pain or need for analgesics, wound infections, and wound seromas between their study groups ${ }^{[21]}$.

The appropriate operative technique involves preserving the cutaneous nerves in the inguinal area to lower the rate and severity of early and late chronic groin pain [CGP] is still in debate. A metaanalysis compared preservation and excision of the ilioinguinal nerve found that preserving the nerve reduces the incidence of local sensitivity loss after the surgery. However, there was no significant difference between nerve excision and preservation regarding CGP or postoperative local numbness[22]. Later studies suggested that these three nerves should be recognized and protected from injury or divided systematically if it was at risk of accidental injury during the procedure[23].

On ultrasound study during the follow-up period, there were no mesh complications in terms of shrinkage, migration, or fragmentation. Migration to a great extent depends on the nature of the mesh and the type of fixation of the mesh. Frequent and deeper penetration of bladder by polypropylene mesh was reported in rats in a study comparing polypropylene, polyglactin, and mixed polypropylene-polyglactin mesh. Erosion of muscularis mucosa of the bladder by polypropylene mesh was found within 14 days ${ }^{[24]}$.

Regarding postoperative pain and the need for analgesia, the current study was based on the hypothesis that suture fixation may potentiate postoperative pain by compression or inadvertent damage to the inguinal nerves. In the current study, there was 3 patient [12\%] in the group [A], and 20 
patients [80\%] in the group [B] had mild pain postoperatively that required increasing the dose of analgesia. While no cases of chronic groin pain were reported in the group $[A]$, there were 3 cases of chronic pain in the group $[B]$ that had mild pain after three months of surgery. There was a statistically significant difference between both groups regarding postoperative pain and need for analgesia [ $<0.001]$, but no statistically significant difference was found regarding chronic pain between both groups. The mean VAS scores in the current study after surgery were 32.7 in the $[A]$ group and 61.2 in the [B] group. There was a significant difference between the study groups regarding mean VAS scores after surgery $[P<0.001]$. The mean time to return to normal activities in the group [A] was 2.51days and 5.97 days in the group $[B]$. Mean time to return to normal activities after surgery was significantly different between study groups [P <0.001].

In the current study, patients in the self-gripping mesh group had consistently lower VAS scores at all follow-up points, and the difference was statistically significant. Analgesic consumption was lower in the self-gripping mesh group with a statistically significant difference. There were significant differences between the study groups regarding mean VAS pain scores at 6 months.

The outcome of early post-operative pain was reported in previous studies. Kingsnorth et al.[10], reported that the visual analog scale pain score compared to baseline was lower in the self-gripping mesh group at discharge and at 7 days; however, the pain increased in the sutured mesh [SM] group at hospital discharge and on 7 days. The difference between the two study groups at both time points was significant $[P=.007$ and .039 , respectively]. Kapischke et al.[25], reported that in the self-gripping mesh [SGM] group, the visual analog scale score was lower significantly than in the SM group [mean 17.9 vs. $32.3 \mathrm{~mm}, \mathrm{P}=.03$ ] on the first postoperative day.

Other studies found that in both groups, the early postoperative pain was statistically similar and claimed that the reasons for early postoperative pain were handling of the hernia sac and surgical trauma, so the use of SGM may not decrease pain incidence ${ }^{[17,26,27]}$. Molegraaft and his colleagues concluded in their study that the sutured mesh has similar results with self-gripping mesh regarding recurrence, chronic postoperative inguinal pain incidence, and foreign body sensation. However, their outcomes measures were heterogenic and long-term results were based on the relatively small patient number[1].

The absence of tension during positioning of the mesh and closure of the prosthesis around the cord can decrease pain created by tension on surrounding tissues and, more particularly, if sutures can be avoided. The grip provides the advantage of obtaining uniform fixation on the whole surface of the mesh that can decrease the like hood of the hernial sac sliding between the prosthesis and the transversalis fascia[28].

Tarchi and his colleagues found in their study that early postoperative pain was mild and may need non-steroidal anti-inflammatory drugs [NSAIDs] to be given for its relief, while chronic postoperative pain with long-term follow up improved even without the use of NSAIDs[18].

Regarding the costs, in the current study, the mean cost of ProGrip mesh was 8 times the mean cost of polypropylene mesh [4508 VS 555 LE]. Bruna et al.[26] stated that although the selfadhesive mesh [€178.69] costed more than the polypropylene mesh [€111.78] and the two 3-0 monofilament sutures [ $€ 1.35$ each] used for fixation, the time saved in surgery-related with their use could equal the overall costs of the procedures. Kapischke et al.[25] reported that the SGM costs more than the comparable mesh of pure polypropylene 2.5 times; however, these increased costs may be compensated by the decreased time of operating room utilization.

Conclusion: The results of the current shortterm study suggest that repair of inguinal hernia with the Lichtenstein technique using self-gripping mesh is an efficient and safe procedure for Egyptian patients. Previous studies conducted in China[13] and Western countries ${ }^{[10]}$ reported similar conclusions.

The current study has some limitations, the small number of patients and the short period of 
follow up. Longer follow-up periods may be required to evaluate the long-term risk of recurrence.

\section{Financial and Non-financial Relationships and Activities of Interest}

None

\section{REFERENCES}

1. Molegraaft M, Kaufman R, Lange J. Comparison of selfgripping mesh and sutured mesh in open inguinal hernia repair: A meta-analysis of long-term results. Surgery. 2018 ;163[2]:351-60. doi: 10.1016/j.surg.2017.08.003.

2. van Steensel S, van Vugt LK, Al Omar AK, et al. Metaanalysis of postoperative pain using non-sutured or sutured single-layer open mesh repair for inguinal hernia. BJS Open. 2019 Jun; 3[3]: 260-273. doi: 10.1002/bjs5.50139.

3. Emile SH, Elfeki $H$. Desarda's technique versus Lichtenstein technique for the treatment of primary inguinal hernia: a systematic review and meta-analysis of randomized controlled trials. Hernia. 2018 Jun;22[3]:385-395. doi: 10.1007/s10029-017-1666-z.

4. Zhang $\mathrm{C}$, Li F, Zhang H, Zhong W, Shi D, Zhao Y. Selfgripping versus sutured mesh for inguinal hernia repair: a systematic review and meta-analysis of current literature. J Surg Res. 2013 Dec;185[2]:653-60. doi: 10.1016/j.jss.2013.07.035.

5. Hawker GA, Mian S, Kendzerska T, French M. Measures of adult pain: Visual Analog Scale for Pain [VAS Pain], Numeric Rating Scale for Pain [NRS Pain], McGill Pain Questionnaire [MPQ], Short-Form McGill Pain Questionnaire [SF-MPQ], Chronic Pain Grade Scale [CPGS], Short Form-36 Bodily Pain Scale [SF-36 BPS], and Measure of Intermittent and Constant Osteoarthritis Pain [ICOAP]. Arthritis Care Res. 2011 Nov;63 Suppl 11:S240-52. doi: 10. 1002/acr.20543.

6. Coda A, Lamberti R, Martorana S. Classification of prosthetics used in hernia repair based on weight and biomaterial. Hernia. 2012 Feb;16[1]:9-20. doi: 10.1007/ s10029-011-0868-z.

7. Jensen EK, Ringsted TK, Bischoff JM, et al. A national center for persistent severe pain after groin hernia repair: Five-year prospective data. Medicine [Baltimore]. 2019 Aug; 98 [33]: e16600. doi: 10.1097/MD. 0000000000016600 .

8. Kaya A, Şahin ST, Kaya Y, et al. Comparison of prolene and progrip meshes in inguinal hernia repair in terms of post-operative pain, limitation of movement and quality of life. Turk J Surg 2020 Mar 18;36[1]:48-52. doi: 10.5578/turkjsurg. 4451.

9. Verhagen T, Zwaans W, Loos M, Charbon J, Scheltinga $M$, Roumen R. Randomized clinical trial comparing self- gripping mesh with a standard polypropylene mesh for open inguinal hernia repair. Br J Surg. 2016 Jun; 103 [7]: 812-8. doi: 10.1002/bjs.10178.

10. Kingsnorth A, Gingell-Littlejohn M, Nienhuijs S, Schüle S, Appel P, Ziprin P. Randomized controlled multicenter international clinical trial of self-gripping Parietex ${ }^{\mathrm{TM}}$ ProGrip ${ }^{\mathrm{TM}}$ polyester mesh versus lightweight polypropylene mesh in open inguinal hernia repair: interim results at 3 months. Hernia. 2012 Jun;16[3]:287-94. doi: 10.1007/s10029-012-0900-y.

11. Maharaul H, Shory V, Joshi S. Comparison of pro-grip mesh $\mathrm{v} / \mathrm{s}$ conventional mesh in Lichtenstein inguinal hernia repair. Inter J Biomed Res 2016;7[10]:738-42.

12. Zwaans WAR, Verhagen $T$, Wouters L, Loos MJA, Roumen RMH, Scheltinga MRM. Groin Pain Characteristics and Recurrence Rates: Three-year Results of a Randomized Controlled Trial Comparing Self-gripping Progrip Mesh and Sutured Polypropylene Mesh for Open Inguinal Hernia Repair. Ann Surg. 2018 Jun; 267 [6]: 1028-1033. doi: 10.1097/SLA. 0000000000002331.

13. Wang $Y$, Zhang $X$. Short-term results of open inguinal hernia repair with self-gripping Parietex ProGrip mesh in China: A retrospective study of 90 cases. Asian J Surg. 2016; 39[4]:218-24. doi: 10.1016/j.asjsur.2015.05.001.

14. Batabyal P, Haddad LR, Samra SJ, et al. Inguinal hernia repair with Parietex Progrip mesh causes minimal discomfort and allows early return to normal activities. Am J Surg. 2016 Jan;211[1]:24-30. doi: 10.1016/ j. amjsurg.2015.04.019.

15. Jorgensen LN, Sommer T, Assaadzadeh S, Strand L, Dorfelt A, Hensler M. Danish Multicentre DANGRIP Study Group. Randomized clinical trial of self-gripping mesh versus sutured mesh for Lichtenstein hernia repair. Br J Surg. 2013;100[4]:474-81. doi: 10.1002/ bjs.9006.

16. Lockhart K, Dunn D, Teo S, Ng JY, Dhillon M, Teo E, van Driel ML Mesh versus non-mesh for inguinal and femoral hernia repair. Cochrane Database Syst Rev. 2018 Sep 13;9[9]:CD011517. doi: 10.1002/14651858. CD011517.pub2.

17. Anadol AZ, Akin M, Kurukahvecioglu O, Tezel E, Ersoy E. A prospective comparative study of the efficacy of conventional Lichtenstein versus self-adhesive mesh repair for inguinal hernia. Surg Today. 2011; 41 [11]: 1498-503. doi: 10.1007/s00595-011-4545-8.

18. Tarchi P, Cosola D, Germani P, Troian M, Manzini De $N$. Self-adhesive mesh for Lichtenstein inguinal hernia repair. Experience of a single center. Minerva Chir. 2014 Jun;69[3]:167-76. PMID: 24736447.

19. Lin H, Zhuang Z, Ma T, Sun X, Huang X, Li Y. A metaanalysis of randomized control trials assessing mesh fixation with glue versus suture in Lichtenstein inguinal 
hernia repair. Medicine [Baltimore]. 2018 Apr;97 [14]: e0227. doi: 10.1097/MD.0000000000010227.

20. Sun $P$, Cheng $X$, Deng $S$, Hu Q, Sun $Y$, Zheng Q. Mesh fixation with glue versus suture for chronic pain and recurrence in Lichtenstein inguinal hernioplasty. Cochrane Database Syst Rev. 2017 Feb 7;2[2]: CD010814. doi: 10.1002/14651858.CD010814.pub2.

21. Rönkä K, Vironen J, Kössi J, Hulmi T, Silvasti S, Hakala $T$ et al. Randomized multi-center trial comparing glue fixation, self-gripping mesh, and suture fixation of mesh in Lichtenstein hernia repair [Finn Mesh Study]. Ann Surg. 2015 Nov;262[5]:714-20. doi: 10.1097/SLA. 0000000000001458.

22. Weyhe D, Belyaev O, Mu€ller C, Meurer K, Bauer KH, Papapostolou $G$ et al. Improving outcomes in hernia repair by the use of light meshes- a comparison of different implant constructions based on a critical appraisal of the literature. World J Surg. 2007 Jan;31[1]:234-44. doi: 10.1007/s00268-006-0123-4.

23. Caliskan K, Nursal TZ, Caliskan E, Parlakgumus A, Yildirim $S$, Noyan T. A method for the reduction of chronic pain after tension-free repair of inguinal hernia: iliohypogastric neurectomy and subcutaneous transposition of the spermatic cord. Hernia. 2010 Feb;14[1]:51-5. doi: 10.1007/s10029-009-0571-5.
24. Cunningham HB, Weis JJ, Taveras LR, Huerta S Mesh migration following abdominal hernia repair: a comprehensive review. Hernia. 2019 Apr;23[2]:235-243. DOl: 10.3205/iprs000135.

25. Kapischke M, Schulze $H$, Caliebe A. Self-fixating mesh for the Lichtenstein procedure--a prestudy. Langenbecks Arch Surg. 2010 Apr;395[4]:317-22. doi: 10.1007/s00423-010-0597-2.

26. Bruna Esteban M, Cantos Pallare M, Artigues Sanchez De Rojas E. Use of adhesive mesh in hernioplasty compared to the conventional technique. Results of a randomised prospective study. Cir Esp. 2010 Oct;88 [4]:253-8. Spanish. doi: 10.1016/j.ciresp.2010.06.008.

27. Pierides $G$, Scheinin $T$, Remes V, Hermunen $K$, Vironen $J$. Randomized comparison of self-fixating and sutured mesh in open inguinal hernia repair. Br J Surg. 2012 May;99[5]:630-6. doi: 10.1002/bjs.8705.

28. Konschake $M$, Zwierzina M, Moriggl B, Függer R, Mayer F, Brunner W, Schmid T, Chen DC, Fortelny R The inguinal region revisited: the surgical point of view: An anatomical-surgical mapping and sonographic approach regarding postoperative chronic groin pain following open hernia repair. Hernia. 2020 Aug;24 [4]: 883-894. doi: 10.1007/s10029-019-02070-z. 


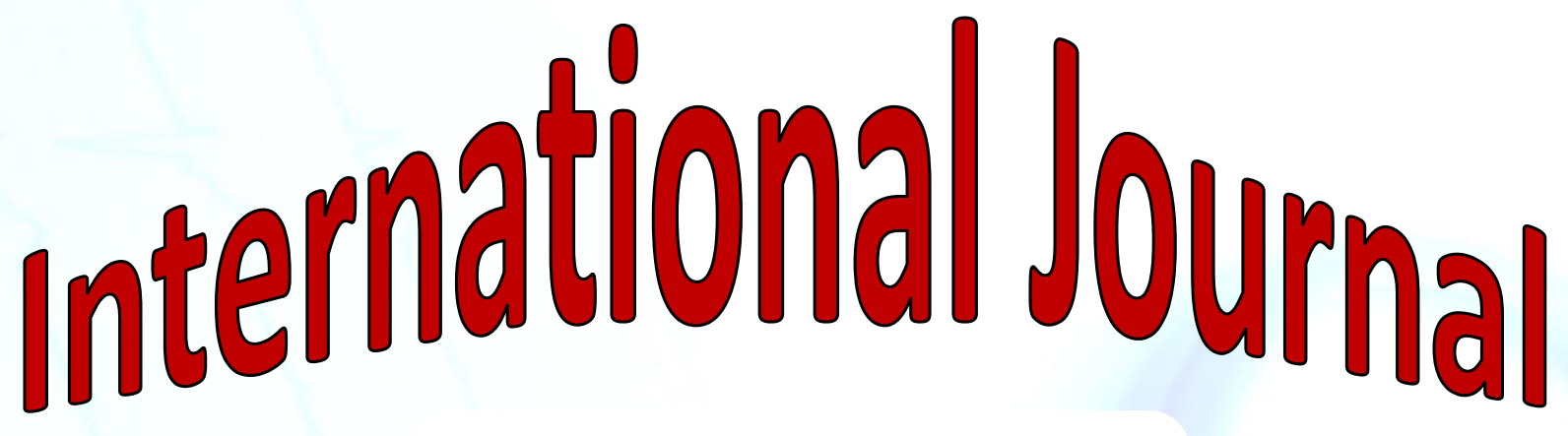

https://ijma.journals.ekb.eg/

Print ISSN: 2636-4174

Online ISSN: 2682-3780

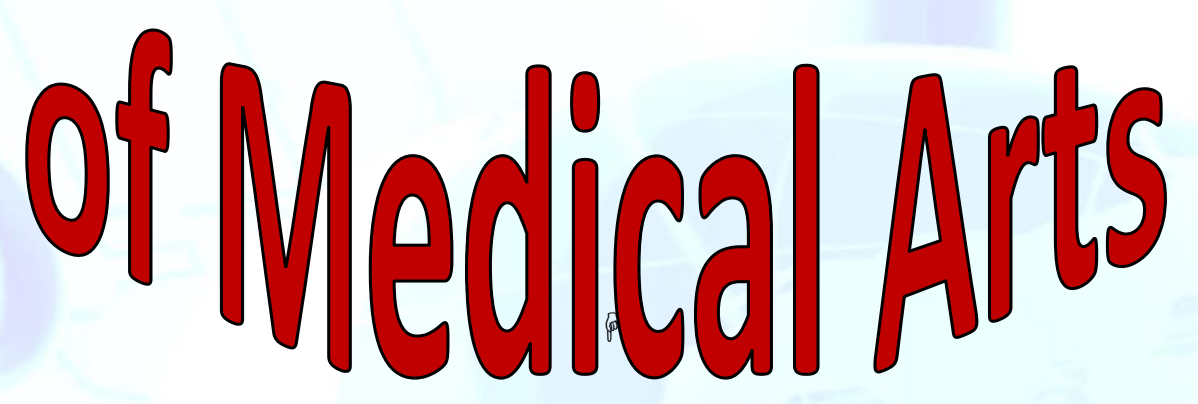

